\title{
Information theoretical view of QCD effective model with heavy quarks
}

\author{
Kouji Kashiwa $\odot^{1, *}$ and Hiroaki Kouno ${ }^{2, \dagger}$ \\ ${ }^{1}$ Fukuoka Institute of Technology, Wajiro, Fukuoka 811-0295, Japan \\ ${ }^{2}$ Department of Physics, Saga University, Saga 840-8502, Japan
}

(Received 2 October 2020; accepted 9 December 2020; published 12 January 2021)

\begin{abstract}
To understand the phase transition phenomena, information theoretical approaches can pick up some important properties of the phenomena based on the probability distribution. In this paper, we show information theoretical aspects of the three-dimensional three-state Potts model with the external field which is corresponded to the QCD effective model with heavy quarks. The transfer mutual information which represents the information flow of two spin variables is numerically estimated based on the Markovchain Monte Carlo method. The transfer mutual information has the peak near the confinementdeconfinement transition, and it may be used to detect the precursors of the transition. Since the transfer mutual information still has the peak even if the Polyakov-loop changes continuously and smoothly, we may pick up some aspects of the confinement-deconfinement nature from the information flow properties. Particularly, the transfer mutual information shows the significantly different behavior below and above the Roberge-Weiss endpoint existed in the pure imaginary chemical potential region, which may indicate the system change by the confinement-deconfinement transition.
\end{abstract}

DOI: 10.1103/PhysRevD.103.014014

\section{INTRODUCTION}

Understanding the confinement-deconfinement transition at finite temperature $(T)$ and chemical potential $(\mu)$ in quantum chromodynamics (QCD) is one of the important and interesting subjects in elementary particle, nuclear, and hadron physics and astrophysics. In the ordinary understanding of the confinement-deconfinement transition at finite temperature in QCD with a vanishing chemical potential, there is no "phase transition," and there is a crossover. In the confinement-deconfinement crossover, there are no singularities in the local order-parameters and also the thermodynamic quantities. However, it has been recently discussed in Ref. [1] that the confinement and deconfinement states at zero temperature can be clarified via the topological order [2], and then it is not necessary that local order-parameters exist and several observables show singular behaviors for the confinement-deconfinement transition. The analogy of the topological order has been applied to the thermal QCD by employing the imaginary chemical potential, $\mu=\left(0, \mu_{\mathrm{I}}\right)$, and then it is expected that confinement-deconfinement transition may be determined from the

\footnotetext{
kashiwa@ fit.ac.jp

†kounoh@cc.saga-u.ac.jp
}

Published by the American Physical Society under the terms of the Creative Commons Attribution 4.0 International license. Further distribution of this work must maintain attribution to the author(s) and the published article's title, journal citation, and DOI. Funded by SCOAP. topological viewpoint [3-5]; see also Ref. [6] for the pioneering work with the imaginary chemical potential and Ref. [7] for the review of the imaginary chemical potential. In the case of the chiral symmetry breaking which is another important feature of QCD, one interesting investigation from the information theoretical view was done by using the thermodynamic geometry $[8,9]$. These studies indicate that we need several viewpoints to correctly understand the confinement-deconfinement nature of QCD.

In this study, we investigate the confinementdeconfinement transition from the information theoretical viewpoints; we discuss the transition directly from the configurations generated by using the Markov-chain Monte Carlo (MCMC) method. Particularly, we use the transfer mutual information and the Kullback-Leibler divergence [10] as the information measure; for example, see Ref. [11] for the application of the Kullback-Leibler divergence to the Polyakov-loop extended Nambu-JonaLasinio model [12]. It is well known that the mutual information should have the peak at the intermediate ordered region [13]: the mutual information becomes small in the highly disordered state because the system elements are almost independent of each other. In comparison, the mutual information also becomes small in the highly ordered state because each element only has small indeterminacy for the system. The highly ordered and disordered states in the parameter region are corresponding to the deconfined and confined phases realized in QCD, ideally. In other words, QCD has the confinementdeconfinement crossover, but we are interested in whether 
the topological nontrivial structure exists or not in certain parameter spaces. There is the possibility that the system does not show the thermodynamics singularities like as the topological order, but some qualitative differences exist.

There are several discussions on the center clustering structure of QCD and some related theories [14-16] which may be related to the confinement-deconfinement transition and topological structure of the system [17]. The center clusters are classified from the topological structure in the space of the phase of the Polyakov-loop (Polyakov-line). In the complex Polyakov-loop plane, the spatial distribution of the local Polyakov-loop can have the wide spread. The distribution of the center cluster depends on the temperature and should have important information about the nature of the confinement-deconfinement nature. Since the mutual information is directly related to the information flow between degrees of freedom (spins in the case of the Potts model) on the nearest-neighbor sites, it is natural to expect that the quantity can be responsible to the center clusters, and also it can be concerned with the interaction properties between degree of freedoms.

The information theoretical quantities such as the Shannon entropy, the mutual information, the cross entropy and the transfer information are widely used in several fields such as financial markets [18,19], human collective decision-making [20], detection of the phase transition $[13,21]$, and so on. There is another interesting information theoretical approach, the persistent homology analysis [22-24], which has been applied to the effective Polyakovline model [17], which has a close relationship with the Potts model and also QCD; see Ref. [25] for recent interesting criteria for the classification of the confinement-deconfinement crossover. The persistent homology is recently applied to the condensed matter physics, and then it has been reported that the hidden order such as spin nematic ordering and spin liquids can be clarified from the persistent homology analysis [26]. Therefore, there is interest in how we could utilize the information theoretic quantity to investigate the mysterious confinementdeconfinement nature of QCD. As a first step to attempt the information theoretical approach to QCD, we start from the QCD effective model with heavy quarks: Actually, the three-dimensional three-state Potts model with the external field is employed in this study because the pure $S U(3)$ Yang-Mills theory has the spontaneous $\mathbb{Z}_{3}$ symmetry breaking and this nature is included in the Potts model; for example, see Ref. [27] for the review of the model and Refs. [28-30] for the relationship with QCD. Other interesting studies of the Potts model which are relevant to QCD properties are shown in Refs. [31-37]. Then, the heavy quark contribution can be mapped to the external field which is composed of the quark mass $(M)$ and the chemical potential $(\mu)$.

In this study, we investigate the behavior of the Kullback-Libeler divergence and the transfer mutual information which represent the information flow in the Markov process and nearest neighbor sites within the several situations by varying the coupling constant, the quark mass and the chemical potential. Then, we can investigate some information theoretical aspects of the confinementdeconfinement nature. In this study, the case of vanishing quark contributions, the pure imaginary chemical potential region and also the real chemical potential region are investigated.

This article is organized as follows. In Sec. II, we explain the formulation of the transfer mutual information and the Kullback-Leibler divergence. The three-dimensional threestate Potts model and its setup are explained in Sec. III. Numerical results are shown in Sec. IV. Section V is devoted to the summary.

\section{TRANSFER MUTUAL INFORMATION AND KULLBACK-LEIBLER DIVERGENCE}

In this section, we explain some entropic quantities proposed in the (classical) information theory. Also, we propose a new quantity which is the so-called transfer mutual information. The hidden central theme of this section is how we can evaluate those quantities within the Monte Carlo method.

\section{A. Mutual information}

The mutual information is defined as

$$
I(A ; B):=H(A)-H(A \mid B),
$$

where $H(X)$ is the Shannon (information) entropy and $H(X \mid Y)$ means the conditional entropy with random variables, $X$ and $Y$. The entropies are constructed by using the probability distributions, $0 \leq p(\mathbf{s}) \leq 1$ with sites $\mathbf{s}$. For example, in the Ising (three-state Potts) model, each site can have 2 degrees of freedom, up and down (3 degrees of freedom). It should be noted that it is difficult to prepare the probability distribution because we need the Monte Carlo method to perform the integration in the complicated theory, particularly in the quantum field theory.

\section{B. Markov process}

Unfortunately, we cannot easily prepare the probability distribution itself by using the Monte Carlo method because we cannot calculate the partition function itself via the simple Monte Carlo method. (It should be noted that the partition function can be estimated by using more complicated algorithms; see Refs. [38-40].) In the case of the two-dimensional kinetic Ising model, we can access the analytic results of the mutual information, but not in the present model. However, we can prepare the probability distribution (ratio) by considering the stochastic process, $X(t)$ and $Y(t)$ with the fictitious time $0 \leq t \in \mathbb{Z}$. When the probability distribution obeys the Boltzmann 
distribution and the detailed valance at stationary is manifested, we have

$$
P_{t-1 \rightarrow t}\left(s_{i}^{(t)}\right)=p\left(s_{i}^{(t)} \mid s_{i}^{(t-1)}\right)=\frac{p\left(s_{i}^{(t)}, s_{i}^{(t-1)}\right)}{p\left(s_{i}^{(t-1)}\right)},
$$

where $s_{i}^{(t)}$ is the randomly selected spin at site $i$ at the $t$-th step. In the Markov-chain Monte Carlo (MCMC) method in the spin model, the random spin flip on a randomly chosen site is accepted or rejected by using the probability; see Ref. [41]. In the Metropolis method, the probability is given by

$$
P_{t-1 \rightarrow t}^{\text {Metropolis }}\left(s_{i}^{(t)}\right)=\min \left(1, e^{-\beta \Delta E}\right),
$$

where $\Delta E$ is the energy difference between the energy at $t$ and that at $t-1$, and $\beta=1 / T$.

\section{Transfer mutual information}

The transfer entropy operator $[13,19,21,42]$ is defined as

$$
\hat{\mathcal{T}}:=-\sum_{s_{i}, s_{j}} p\left(s_{i}^{(t)} \mid s_{i}^{(k)}, s_{j}^{(l)}\right) \ln \frac{p\left(s_{i}^{(t)} \mid s_{i}^{(k)}, s_{j}^{(l)}\right)}{p\left(s_{i}^{(t)} \mid s_{i}^{(k)}\right)},
$$

where $t, k, l \in \mathbb{Z}$ indicate the time step of the MCMC process. Usually, we take $l=t-1$ which corresponds to the time at the configuration generation and $k=t-1$. Also, $i$ and $j$ are taken so as to the nearest neighbors. With these settings, $\hat{\mathcal{T}}$ is the pairwise mutual information operator and it is denoted as $\hat{\mathcal{T}}_{\text {pw }}$ below.

In this study, we cannot use any analytic expressions of the probability distributions and thus we deform it to be calculable within the MCMC method as

$$
\begin{aligned}
\hat{\mathcal{T}}_{\mathrm{pw}}:= & -\sum_{s_{i}} p\left(s_{i}^{(t)} \mid \mathbf{s}^{(t-1)}\right) \ln p\left(s_{i}^{(t)} \mid \mathbf{s}^{(t-1)}\right) \\
& -\sum_{s_{j}} p\left(s_{j}^{(t)} \mid \mathbf{s}^{(t-1)}\right) \ln p\left(s_{j}^{(t)} \mid \mathbf{s}^{(t-1)}\right) \\
& +\sum_{s_{i}, s_{j}} p\left(s_{i}^{(t)}, s_{j}^{(t)} \mid \mathbf{s}^{(t-1)}\right) \ln p\left(s_{i}^{(t)}, s_{j}^{(t)} \mid \mathbf{s}^{(t-1)}\right),
\end{aligned}
$$

where $p\left(s_{i}^{(t)} \mid \mathbf{s}^{(t-1)}\right)$ means the probability that the spin at site $s_{i}$ is flipped and $p\left(s_{i}^{(t)}, s_{j}^{(t)} \mid \mathbf{s}^{(t-1)}\right)$ does the probability that the spin at site $s_{i}$ and the nearest neighbor site $s_{j}$ are flipped. The subscript $t-1$ means the (fictitious) time where the configuration is generated. This quantity can be calculated by using the MCMC method without the analytic expression of the probability distribution. Thus, we call it as the transfer mutual information. When we evaluate the expectation value of $\hat{\mathcal{T}}_{\text {pw }}$, we should take into account all possible pairs of lattice nearest neighbors as

$$
\mathcal{T}_{\mathrm{pw}}=\frac{1}{3 V} \sum_{\langle i j\rangle}\left\langle\hat{\mathcal{T}}_{\mathrm{pw}}\right\rangle,
$$

where $V$ is the spatial volume of the system and $3 V$ is the number of the independent pairs of nearest neighbors sites; the lattice spacing is set to 1 below. It should be noted that expectation values $\mathcal{T}_{\mathrm{pw}}$ and $D_{\mathrm{KL}}$ are estimated with the Boltzmann weight based on the Metropolis method.

The spin flip is imposed to each configuration after the configuration generation process. This means that the measurement of $\mathcal{T}_{\mathrm{pw}}$ and $D_{\mathrm{KL}}$ requires additional computing cost which depends on properties of considering interaction; if one spin interacts with all other spins, the additional computing time is proportional to the total number of spins comparing with the measurement of the spin average. From the viewpoint of the numerical cost, those information theoretical quantities do not have the advantage, but the main purpose of this study is to qualitatively know how those quantities can pick up the system change. Of course, the transfer mutual information uses the MCMC probability distribution, and thus it may depend on the scheme, quantitatively. It should be noted that the transfer mutual information contains the information flow of the Markov process and also that from the nearest neighbor sites. In the two-dimensional kinetic Ising model, it is known that the mutual information and the transfer entropy share almost similar properties [13] and thus the present transfer mutual information can be considered as the acceptable quantity for our purpose. From the mutual information with the above setting, we can treat not only the global structure of the system, but also the local spatial structure of the degree of freedom which is the spin in the Potts model. Therefore, it is expected that the mutual information is a good quantity to investigate the system which has the nontrivial spatial structure.

There is the possibility that we can use some more different probabilities. Below, we consider QCD as an example. One choice is using the multiplicity distribution defined as

$$
g_{n}=\mathcal{Z}_{n} \xi^{n}
$$

where $\mathcal{Z}_{n}$ means the canonical partition function with the quark number $n$ and $\xi$ standing for the fugacity. This quantity can bridge the experimental data and the numerical simulation even in QCD [43], and it is nothing but the probability distribution of the net quark number. To make it as the probability distribution, $p_{n}$, we should consider

$$
p_{n}=\frac{g_{n}}{\mathcal{N}},
$$


where $\mathcal{N}$ is the normalization factor which corresponds to the grand-canonical partition function itself. With the probability, there is no scheme dependence, but it needs a heavy numerical cost to prepare the canonical partition function, which requires the accurate Fourier transformation; see Ref. [44] as an example. In addition, by using the probability distribution (8), we can evaluate the information flow between each canonical sector, but not that between the degree of freedoms. Therefore, we employ the probability distribution (3) that appeared in the MCMC process in this study.

\section{Kullback-Leibler divergence}

In the information theory, we have another entropy which is called the relative entropy. A part of the relative entropy is so called the Kullback-Leibler divergence, and the operator in the present situation with the Markov process can be expressed as

$$
\hat{D}_{\mathrm{KL}}=\sum_{i} p\left(s_{i}^{(t)} \mid \mathbf{s}^{(t-1)}\right) \ln p\left(s_{i}^{(t)} \mid \mathbf{s}^{(t-1)}\right) .
$$

It should be noted that this expression seems different from the standard Kullback-Leibler divergence, but the present $p\left(s_{i}^{(t)} \mid \mathbf{s}^{(t-1)}\right)$ is the conditional probability in the operator and thus is should be suitable to consider the following expectation values with the Boltzmann weight $p\left(s_{i}^{(t-1)}\right)$. The expectation value of the Kullback-Leibler divergence operator is

$D_{\mathrm{KL}}=\frac{1}{3 V}\left\langle\hat{D}_{\mathrm{KL}}\right\rangle=\frac{1}{3 V} \sum_{\mathbf{s}} \sum_{i} p\left(s_{i}^{(t)}\right) \ln p\left(s_{i}^{(t)} \mid \mathbf{s}^{(t-1)}\right)$,

and it represents how similar the probability distributions are. Thus, the transfer mutual information and the Kullback-Leibler divergence show different aspects of the information about the system. It should be noted that when we replace the probability distributions in $D_{\mathrm{KL}}$ with the conditional probabilities, we can obtain the mutual information.

For the reader's convenience, here we summarize the general properties of the Kullback-Leibler divergence. In general, the Kullback-Leibler divergence is not bounded as above; if the two probability distributions are not overlapping completely $\left[\forall x, p_{1}(x) \neq p_{2}(x)\right]$, the KullbackLeibler divergence becomes $\infty$. On the other hand, the Kullback-Leibler divergence becomes 0 if the two probability distributions are the same. To make the quantity finite in the case with $p_{1}(x) \neq p_{2}(x)$, we may use the JensenShannon divergence because it does not diverge, $0 \leq D_{\text {JS }} \leq$ $\ln 2$ [45]. However, the Kullback-Leibler divergence calculated in this paper (10) is not diverged even in the thermodynamic limit as explained after Eq. (13).
It should be noted that the Kullback-Leibler divergence is related to the Fischer's information matrix. With the Taylor expansion, the Kullback-Leibler divergence can be expressed as

$$
D_{\mathrm{KL}}=\frac{1}{2} \sum_{s} \sum_{t} I_{s t} \delta \lambda_{s} \delta \lambda_{t}
$$

where $\lambda_{s}, \lambda_{t}$ mean continuous parameters such as $T$ and $\mu$, and $I_{s t}$ is called the Fischer's information matrix which is the second-order derivative of $D_{\mathrm{KL}}$ by $\lambda_{s}$ and $\lambda_{t}$. When we replace $D_{\mathrm{KL}}$ with the free energy, it corresponds to the thermodynamic geometry recently discussed in QCD [8,9]. Since the Kullback-Leibler divergence with the MCMC probability should have the thermodynamic information of the theory because the probability distribution is directly related to the thermodynamics, it is interesting to investigate the Kullback-Leibler divergence at finite $T$ and $\mu$. Actually, the deep relation between the Kullback-Leibler divergence and the phase transition is a known fact in statistical mechanics.

\section{QCD EFFECTIVE MODEL WITH HEAVY QUARKS}

In this study, we employ the three-dimensional threestate Potts model as the QCD effective model with the heavy quarks [28-30]. The Potts model energy is

$$
E=-\kappa \sum_{\mathbf{x}, \mathbf{i}} \delta_{\Phi_{\mathbf{x}}, \Phi_{\mathbf{x}+\mathbf{i}}}+\sum_{\mathbf{x}}\left(h_{+} \Phi_{\mathbf{x}}+h_{-} \bar{\Phi}_{\mathbf{x}}\right),
$$

where $\mathbf{i}$ means the unit vector in the three-dimensional space, $\kappa$ means the coupling constant, $h_{ \pm}$denotes the external field and $\Phi_{\mathbf{x}}\left(\bar{\Phi}_{\mathbf{x}}\right)$ is the $\mathbb{Z}_{3}$ values (its conjugate) on each site; it corresponds to the Polyakov-loop in QCD. It should be noted that the energy difference for the single spin flip $\left(\Delta E_{1}\right)$ does not depends on the system volume, and thus the original probability and the single-spin flipped probability can have the overlap. For the reader's convenience, we here show the energy difference for the single spin flip at one site $\mathbf{x}$,

$$
\begin{aligned}
\Delta E_{1, \mathbf{x}}= & -\kappa \sum_{\mathbf{i}}\left(\delta_{\Phi_{\mathbf{x}}, \Phi_{\mathbf{x}+\mathbf{i}}}-\delta_{\Phi_{\mathbf{x}}^{\prime}, \Phi_{\mathbf{x}+\mathbf{i}}^{\prime}}\right) \\
& +\left[h_{+}\left(\Phi_{\mathbf{x}}-\Phi_{\mathbf{x}}^{\prime}\right)+h_{-}\left(\bar{\Phi}_{\mathbf{x}}-\bar{\Phi}_{\mathbf{x}}^{\prime}\right)\right],
\end{aligned}
$$

where $\Phi^{\prime}\left(\bar{\Phi}^{\prime}\right)$ means the spin flipped $\mathbb{Z}_{3}$ values (its conjugate). For the single-spin flip case at one site, almost all contributions are canceled out because we only take the sum of nearest neighbor sites of $\mathbf{x}$ and then $\Delta E_{1, \mathrm{x}}$ appears as $e^{-\beta \Delta E_{1, \mathrm{x}}}$ in Eq. (10). Therefore, $D_{\mathrm{KL}}$ does not diverge even in the thermodynamic limit at finite $\beta$. In other words, in the present paper, we calculate $D_{\mathrm{KL}}$ from the 
corresponding one spin-flipped configuration for each configuration, and thus we do not encounter the divergence.

The external fields are expressed with the quark mass and the chemical potential as

$$
h_{ \pm}=e^{-\beta(M \mp \mu)},
$$

which is induced from the fermion determinant in QCD partition function; see the Appendix for details. If the quark mass is sufficiently heavy in QCD, the three-dimensional three-state Potts model with the external field can be treated as the effective model of QCD. Recently, the extension of the Potts model has been done by considering the $\mathbb{Z}_{3}$ symmetrization and has been used to investigate the sign problem [46]; see Ref. [47] for details of the sign problem. This means that the analysis of the Potts model is still important to understand some QCD properties.

At nonzero real $\mu$, Eq. (12) is no longer real at each MCMC process, and then we cannot consider that $e^{-\beta H}$ is the probability distribution which leads the Boltzmann distribution as the stationary distribution. One choice to overcome the difficulty is using the phase quenched probability distribution in the configuration averaging procedure and employing the reweighting. However, if the average phase factor becomes smaller, it is very difficult to obtain reliable results. In this paper, thus, we consider the small real chemical potential because the sign problem is not so serious. Also, we introduce the imaginary chemical potential because Eq. (12) goes back to real values.

To calculate $\mathcal{T}_{\mathrm{pw}}$ and $D_{\mathrm{KL}}$, we utilize the MCMC method. We here use the Metropolis method to perform the spin flip process, and then we generate $10^{4}$ configurations, analyzing each 100 updation after the thermalization. Simulations are performed with $V=6^{3}, 8^{3}$ and $10^{3}$. We generate $10^{4}$ configurations, and errors are estimated by using the JackKnife method with the bin size $N_{\text {bin }}=10$. It should be noted that in the case of $\mu / T=9$ in Fig. 2, the statistic error tend to be somewhat large because of the sign problem, and thus we generate $10^{6}$ configurations with $N_{\text {bin }}=100$. For the random number generation, we employ the Mersenne twister algorithm [48].

For the reader's convenience, we here show the CPU time for the one configuration generation $\left(t_{\text {conf }}\right)$ and the measurement with one configuration $\left(t_{m}\right)$ where $m$ means the $\Phi, D_{\mathrm{KL}}$ and $\mathcal{T}_{\mathrm{pw}}$ with the setting, $\beta=1, M=10$, $\kappa=0.02, h_{ \pm}=0$ and $V=6^{3}$, as an example: the CPU times in seconds up to three significant figures are $t_{\text {conf }}=$ $1.72 \times 10^{-4} \times N_{\text {step }}$ where $N_{\text {step }}-1$ means the number of discarded data between the former and the latter configuration, $t_{\Phi}=2.73 \times 10^{-4}, t_{D_{\mathrm{KL}}}=1.72 \times 10^{-2}$ and $t_{\mathcal{T}_{\mathrm{pw}}}=2.38 \times 10^{-2}$. Above times are obtained with the Intel Core i7-7820X CPU @ $3.60 \mathrm{GHz} \times 16$. Therefore, the total configuration generations and the measurements basically need the time $t_{\text {conf }} \times\left(N_{\text {conf }} N_{\text {skip }}+N_{\text {ther }}\right)$ and $t_{m} \times N_{\text {conf }}$ where $N_{\text {ther }}$ is discarded data for thermalization, and $N_{\text {conf }}$ means the number of configurations generated in the calculation.

\section{NUMERICAL RESULTS}

We show our numerical results with the real and imaginary external fields and without the external field by using the Monte Carlo method.

\section{A. Vanishing $\boldsymbol{h}_{ \pm}$}

In this situation, we have the exact $\mathbb{Z}_{3}$ symmetry in Eq. (12), and then we always have $\langle\Phi\rangle=0$ in principle; even if the symmetry is spontaneously broken, the configuration averaging procedure leads to $\langle\Phi\rangle=0$; for example, see Ref. [49]. It should be noted that we can take the absolute value of the spatially averaged $\Phi$ before taking the configuration average, and then the expectation value still remains nonzero; we abbreviate it as $|\Phi|$ below. In this study, we introduce the extremely small external field to make the spin have a unique direction after the spontaneous symmetry breaking. It is the standard procedure to detect the phase transition. Also, we do not take extrapolation to the thermodynamic limit, and thus the Polyakov-loop still has the nonzero value in the confined phase; it is nothing but the finite size artifact.

Figure 1 shows the $\kappa$-dependence of $|\Phi|, \mathcal{T}_{\mathrm{pw}}$ and $D_{\mathrm{KL}}$ at $\beta=1$. The peak exists in the $\mathcal{T}_{\mathrm{pw}}$, and it appears very close to the rapidly changing point of $|\Phi|$ and $D_{\mathrm{KL}}$. This indicates that the transfer mutual information picks up the fluctuation of the system from the spin configuration space. Also, the finite size effects in the transfer mutual information seems
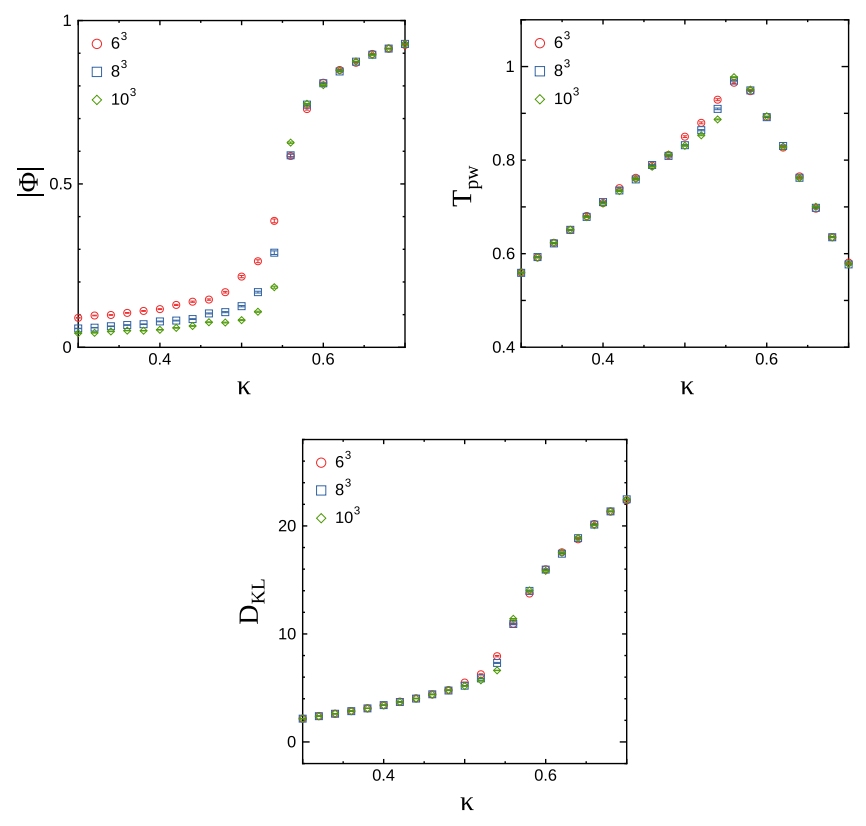

FIG. 1. The $\kappa$-dependence of the $|\Phi|, \mathcal{T}_{\mathrm{pw}}$ and $D_{\mathrm{KL}}$ at $\beta=1$ with $V=6^{3}, 8^{3}$ and $10^{3}$. 
to be smaller than that in the Polyakov-loop. Since the present mutual information is related with the information flow between nearest neighbor sites, the finite size effect is expected to be small.

\section{B. Nonzero real $\mu$}

In this case, the three-dimensional three-state Potts model has the sign problem, and it becomes more serious when the chemical potential becomes larger. To circumvent the problem, we here use the reweighting method [50-53]; we make the probability distribution by using the phase quenched one and the observable quantities are reweighted to make it correctly. Actually, we prepare the MCMC probability by using the absolute value of the original Boltzmann weight, $\left|e^{-\beta H}\right|$, with the phase factor, $e^{-\beta H} /\left|e^{-\beta H}\right|$. However, when the average phase factor which represents the similarity between the original and phase quenched probability distributions becomes smaller, we cannot obtain reliable results, and thus we cannot consider the whole chemical potential region. We here consider $\mu<M$. In the Potts model, there are several modern methods to circumvent the sign problem, but we are interested in the information flow, and thus we still use the standard Metropolis algorithm; for example, see Refs. [37,54] for smart approaches to the sign problem appearing in the Potts model.

Figure 2 shows the $\kappa$-dependence of $|\Phi|, \mathcal{T}_{\mathrm{pw}}$ and $D_{\mathrm{KL}}$ with $\beta=1, M=10$ and $V=6^{3}$. Because of the small average phase factor at finite $\mu$, the error bar becomes large, but we can see that the rapidly changing point of $|\Phi|$ decreases with increasing $\mu$. The smoothing behavior of the
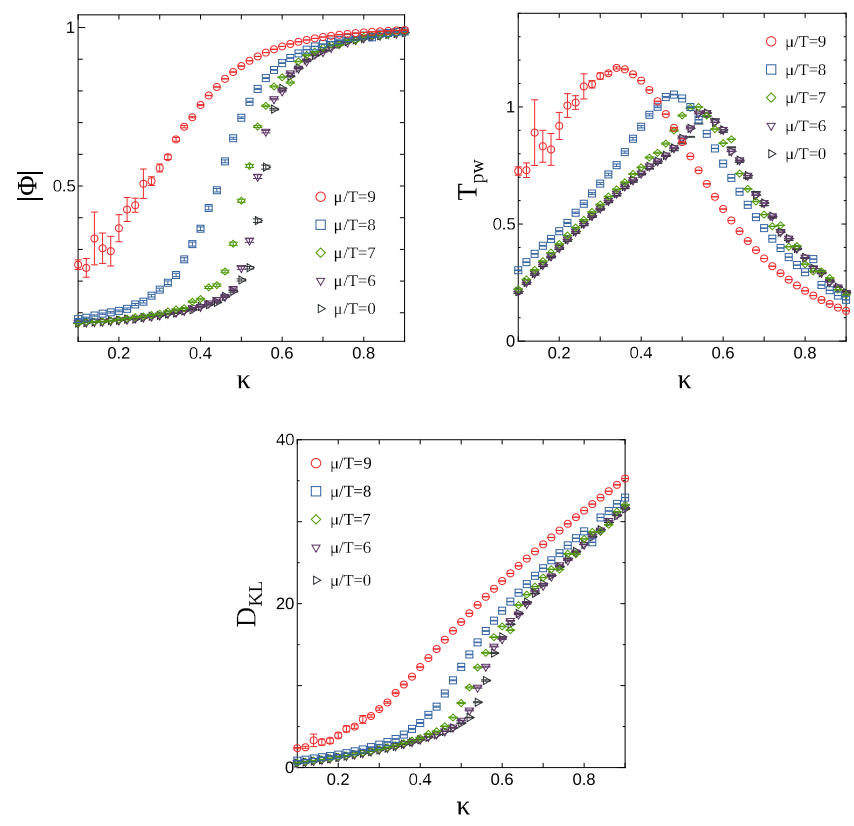

FIG. 2. The $\kappa$-dependence of $|\Phi|, \mathcal{T}_{\mathrm{pw}}$ and $D_{\mathrm{KL}}$ where $\beta=1$ with $M=10$ and $V=6^{3}$, respectively.
Polyakov loop with increasing $\mu / T$ is the opposite behavior compared to the chiral transition in QCD. The KullbackLeibler divergence shows similar behavior with the Polyakov-loop. Interestingly, the peak position of $\mathcal{T}_{\mathrm{pw}}$ shifted to lower $\kappa$ with increasing $\mu$ and the peak strength becomes strong. This strongly indicates that the correlations between nearest neighbor sites is enhanced. Also, the transfer mutual information feels effects of $\mu$ in the confined phase rather than the deconfined phase. This means that the confinement phase has strong information flow coming from fermion effects.

\section{Nonzero pure imaginary $\mu$}

Finally, we consider the pure imaginary chemical potential. The imaginary chemical potential can be interpreted as the flux insertion to the fictitious hole of the time direction in the QCD with the imaginary time formulation. In this case, we do not encounter the sign problem, and also there is the Roberge-Weiss (RW) transition along the $\theta:=\mu /(i T)$ direction in addition to the confinement-deconfinement transition [55-59]. Also, there may be the possibility that we can pick up some information of the confinementdeconfinement transition from this region [3-5]. It should be noted that the following results are the first results which explicitly show the detailed behavior of the Roberge-Weiss periodicity and the transition in the Potts model with the external field.

In the Potts model, spin itself is a more fundamental quantity compared to the Polyakov-loop, and thus we here calculate

$$
\langle\text { Average spin }\rangle=\left\langle\frac{1}{V} \sum_{\mathbf{x}} \operatorname{spin}(\mathbf{x})\right\rangle,
$$

where we assign spins as $0,+1$ and -1 for $\arg (\Phi)=0$, $\arg (\Phi)=2 \pi / 3$ and $\arg (\Phi)=4 \pi / 3$, respectively. The $\theta$-dependence of the average spin with $\beta \kappa=0.4$ and 0.6 with $\beta=0.1$ is shown in the top-left panel of Fig. 3. To clearly see the oscillating behavior, we here take $\beta=0.1$ and $M=10$. We can clearly see that there is the RW transition, if $\kappa$ is sufficiently large where $|\Phi|$ has large value. The top-right and bottom panels of Fig. 3 show the $\theta$-dependence of $\mathcal{T}_{\mathrm{pw}}$ and $D_{\mathrm{KL}}$, respectively. We can see that the transfer mutual information and the KullbackLeibler divergence show the RW periodicity and the tendency of the RW transition although the $\mathbb{Z}_{3}$ symmetry is explicitly broken due to the effect of dynamical quark contribution. Interestingly, the oscillation of the transfer mutual information becomes significantly different behavior below and above the RW endpoint. This indicates that the information flow is different, and it has the additional information about the confinement-deconfinement nature compared to the Polyakov-loop and the Kullback-Leibler divergence. 

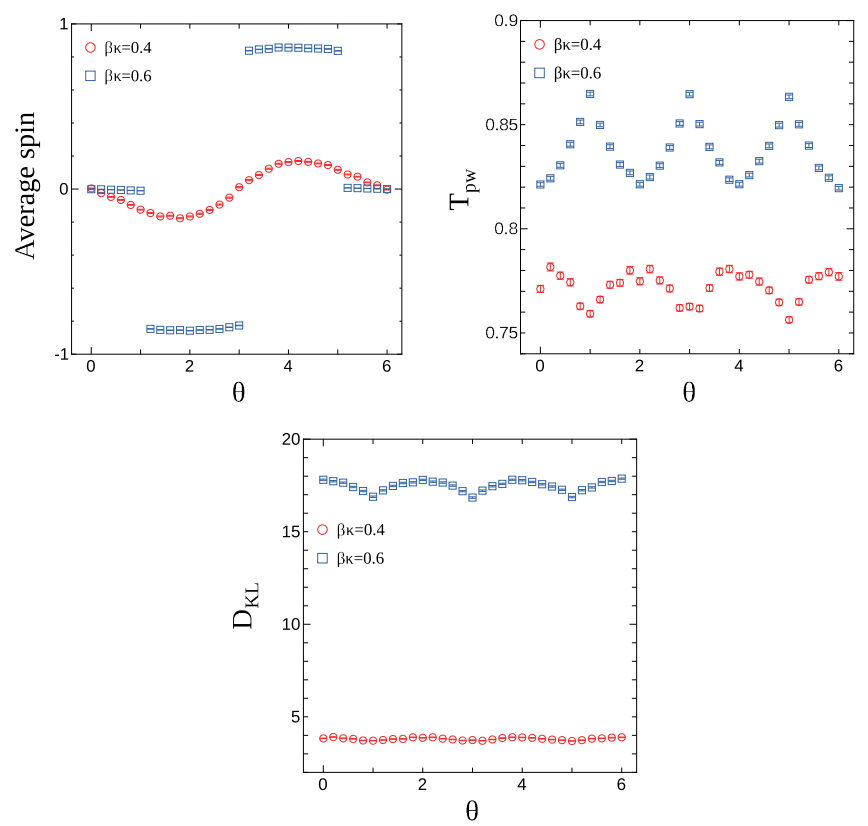

FIG. 3. The $\theta$-dependence of the average spin, $\mathcal{T}_{\mathrm{pw}}$ and $D_{\mathrm{KL}}$ with $V=6^{3}$. The circle and square symbols are results with $\beta \kappa=$ 0.4 and 6 with $\beta=0.1$ and $M=10$, respectively.

\section{SUMMARY}

In this study, we have investigated the information theoretical aspects of the confinement-deconfinement nature in the QCD effective model with heavy quarks; the three-dimensional three-state Potts model is employed. We have considered the transfer mutual information which represents the information flow in the Markov process and the lattice nearest neighbor sites in the case of the pure gauge limit, the finite imaginary chemical potential region and the finite real chemical potential region to understand the confinement-deconfinement transition. The transfer mutual information can pick up the confinementdeconfinement transition of the system, and then it has the peak around the transition point unlike the KullbackLeibler divergence which has the rapidly changing point around the point. Also, it can clarify the Roberge-Weiss transition and its endpoint which is important to understand QCD properties at finite density.

At finite real chemical potential, we employ the reweighting method and calculate the Polyakov-loop, the transfer mutual information and the Kullback-Leibler divergence. We have found that the value of $\kappa$ at the peak position of the transfer mutual information decreases with increasing the real chemical potential. Also, the transfer mutual information still has the peak position if the system indicates the crossover from the Polyakov-loop behavior. This means that there is the region where the information transfer is still active even when the Polyakov-loop indicates the crossover behavior of the thermal system. It may mean that there is the possibility that the significant system changes which are not characterized by the order parameter of the spontaneous symmetry breaking exist. To clarify it, we may need more studies on the confinementdeconfinement transition from the information theoretical approach.

When we apply the transfer mutual information to QCD, we need greater extension since the degree of freedom in QCD is not the discrete spin but the $S U(3)$ gauge field. However, the field still has the $\mathbb{Z}_{3}$ symmetry in the pure gauge limit, and thus we can define the $\mathbb{Z}_{3}$ spin flip which has the perfect analogy with the spin flip in the three-state Potts model. Therefore, we can calculate the transfer mutual information in QCD by using the $\mathbb{Z}_{3}$ flip (transformation) employing the Metropolis update procedure. We will report it elsewhere. Particularly, QCD has another interesting nature which is the so-called the chiral symmetry breaking, and thus it is interesting how the transfer mutual information feels the phenomena.

\section{ACKNOWLEDGMENTS}

$\mathrm{K} . \mathrm{K}$. is grateful to Y. Kikuchi for discussions on the relation between the Potts model and QCD. We appreciate S. Hawkins for reading the manuscript via the English writing correction service provided from Fukuoka Institute of Technology. This work is supported in part by the Grants-in-Aid for Scientific Research from JSPS (Grants No. 18K03618, No. 19H01898, and No. 20K03974).

\section{APPENDIX: MAPPING OF $m$ AND $\mu$ TO THE EXTERNAL FIELD OF POTTS MODEL}

Based on Ref. [60], we can understand how the Potts model has the relation with QCD, particularly how to map the quark mass and the chemical potential in QCD to the external field in the Potts model.

With the sufficiently heavy quark mass, quarks can be treated as the static quark and then quarks obey the timeevolution equation,

$$
\begin{aligned}
\left(\frac{\partial}{\partial \tau}+i A_{4}+\gamma_{0} m\right) q(\vec{x}, \tau) & =0 \\
\rightarrow\left[\left(\frac{\partial}{\partial \tau}+A_{4}\right)^{2}-m^{2}\right] q(\vec{x}, \tau) & =0,
\end{aligned}
$$

where we consider the Euclidean space-time. To introduce $\mu$, we should replace $A_{4}$ with $A_{4}-i \mu$. The solution of the equation becomes

$$
q(\vec{x}, \tau)=\mathcal{T} \exp \left[i \int_{0}^{\beta} A_{4} d \tau-\beta(m-\mu)\right] q(\vec{x}, 0),
$$

where $\mathcal{T}$ means the time-ordering operator, and we take the solution $\exp (-\beta m)$ instead of $\exp (\beta m)$ to match the pure gauge limit. 
The partition function with static $N_{q}$-quarks and $N_{\bar{q}^{-}}$ antiquarks is

$$
\begin{aligned}
\sum_{|s\rangle}\left\langle s\left|e^{-\beta \mathcal{H}}\right| s\right\rangle & =\operatorname{tr}_{\mathrm{c}}\left[e^{-\beta \mathcal{H}} L\left(\vec{x}_{1}\right) \cdots L\left(\vec{x}_{N_{q}}\right) L^{\dagger}\left(\vec{x}_{1}\right) \cdots L^{\dagger}\left(\vec{x}_{N_{\bar{q}}}\right)\right] \\
& \sim \mathcal{Z}
\end{aligned}
$$

where $|s\rangle$ means the state with static $N_{q^{\text {-quarks }}}$ and $N_{\bar{q}^{-}}$ antiquarks, $\mathcal{H}$ is the pure gauge Hamiltonian which is modeled by the standard Potts model and $L$ means the modified operator of the Polyakov loop defined as

$$
L(\vec{x})=\frac{1}{N_{\mathrm{c}}} \operatorname{tr}_{\mathrm{c}} \mathcal{T} e^{i \int_{0}^{\beta} A_{4} d \tau} e^{-\beta(m-\mu)},
$$

where $\operatorname{tr}_{\mathrm{c}}$ means the trace acting on the color space. From the result, we can read off the effective Hamiltonian for the QCD with heavy quarks which corresponds to the Potts Hamiltonian with the external field; we can understand how the Potts spin correlates with the quark mass and the chemical potential after taking sums of the number of quark and antiquark, $h_{ \pm}=\exp \beta[-(m \mp \mu)]$. For more details except the present mapping, see Ref. [28]. It should be noted that we can understand why the Polyakov-loop is related to the free energy for the single-quark excitation in the static limit and why the relation cannot be manifested with dynamical quarks from the equations.
[1] M. Sato, Phys. Rev. D 77, 045013 (2008).

[2] X. G. Wen, Int. J. Mod. Phys. B 04, 239 (1990).

[3] K. Kashiwa and A. Ohnishi, Phys. Lett. B 750, 282 (2015).

[4] K. Kashiwa and A. Ohnishi, Phys. Rev. D 93, 116002 (2016).

[5] K. Kashiwa and A. Ohnishi, Phys. Lett. B 772, 669 (2017).

[6] N. Weiss, Phys. Rev. D 35, 2495 (1987).

[7] K. Kashiwa, Symmetry 11, 562 (2019).

[8] P. Castorina, D. Lanteri, and S. Mancani, Eur. Phys. J. Plus 135, 43 (2020).

[9] B. Zhang, S.-S. Wan, and M. Ruggieri, Phys. Rev. D 101, 016014 (2020).

[10] S. Kullback and R. A. Leibler, Ann. Math. Stat. 22, 79 (1951).

[11] K. Kashiwa and A. Ohnishi, arXiv:1712.06220.

[12] K. Fukushima, Phys. Lett. B 591, 277 (2004).

[13] L. Barnett, J. T. Lizier, M. Harré, A. K. Seth, and T. Bossomaier, Phys. Rev. Lett. 111, 177203 (2013).

[14] C. Gattringer, Phys. Lett. B 690, 179 (2010).

[15] S. Borsanyi, J. Danzer, Z. Fodor, C. Gattringer, and A. Schmidt, J. Phys. Conf. Ser. 312, 012005 (2011).

[16] G. Endrodi, C. Gattringer, and H.-P. Schadler, Phys. Rev. D 89, 054509 (2014).

[17] T. Hirakida, K. Kashiwa, J. Sugano, J. Takahashi, H. Kouno, and M. Yahiro, Int. J. Mod. Phys. A 35, 2050049 (2020).

[18] M. Harré and T. Bossomaier, Europhys. Lett. 87, 18009 (2009).

[19] Z. Deng, J. Wu, and W. Guo, Phys. Rev. E 90, 063308 (2014).

[20] G. Carbone and I. Giannoccaro, Eur. Phys. J. B 88, 339 (2015).

[21] H. W. Lau and P. Grassberger, Phys. Rev. E 87, 022128 (2013).

[22] H. Edelsbrunner, D. Letscher, and A. Zomorodian, in Proceedings 41st Annual Symposium on Foundations of Computer Science (IEEE, New York, 2000), pp. 454-463, https://ieeexplore.ieee.org/abstract/document/892133.
[23] A. Zomorodian and G. Carlsson, Discrete Comput. Geom. 33, 249 (2005).

[24] T. Nakamura, Y. Hiraoka, A. Hirata, E. G. Escolar, and Y. Nishiura, Nanotechnology 26, 304001 (2015).

[25] K. Fukushima, T. Kojo, and W. Weise, Phys. Rev. D 102, 096017 (2020).

[26] B. Olsthoorn, J. Hellsvik, and A. V. Balatsky, Phys. Rev. Research 2, 043308 (2020).

[27] F.-Y. Wu, Rev. Mod. Phys. 54, 235 (1982).

[28] M. G. Alford, S. Chandrasekharan, J. Cox, and U. J. Wiese, Nucl. Phys. B602, 61 (2001).

[29] S. Kim, P. de Forcrand, S. Kratochvila, and T. Takaishi, Proc. Sci. LAT2005 (2006) 166 [arXiv:hep-lat/0510069], https://pos.sissa.it/020/.

[30] P. de Forcrand and O. Philipsen, Phys. Rev. Lett. 105, 152001 (2010).

[31] M. Fukugita and M. Okawa, Phys. Rev. Lett. 63, 13 (1989).

[32] Y. Delgado Mercado, H. G. Evertz, and C. Gattringer, Comput. Phys. Commun. 183, 1920 (2012).

[33] F. Karsch and S. Stickan, Phys. Lett. B 488, 319 (2000).

[34] F. Karsch, C. Schmidt, and S. Stickan, Comput. Phys. Commun. 147, 451 (2002).

[35] C. Bonati and M. D’Elia, Phys. Rev. D 82, 114515 (2010).

[36] C. Gattringer, D. Göschl, and P. Törek, Nucl. Phys. B956, 115008 (2020).

[37] A. Alexandru, G. Bergner, D. Schaich, and U. Wenger, Phys. Rev. D 97, 114503 (2018).

[38] A. Hasenfratz and D. Toussaint, Nucl. Phys. B371, 539 (1992).

[39] F. Wang and D. Landau, Phys. Rev. Lett. 86, 2050 (2001).

[40] A. Bazavov and B. A. Berg, Phys. Rev. D 75, 094506 (2007).

[41] N. Metropolis, A. W. Rosenbluth, M. N. Rosenbluth, A. H. Teller, and E. Teller, J. Chem. Phys. 21, 1087 (1953).

[42] T. Schreiber, Phys. Rev. Lett. 85, 461 (2000).

[43] A. Nakamura and K. Nagata, Prog. Theor. Exp. Phys. 2016, 033D01 (2016). 
[44] R. Fukuda, A. Nakamura, and S. Oka, Phys. Rev. D 93, 094508 (2016).

[45] J. Lin, IEEE Trans. Inf. Theory 37, 145 (1991).

[46] T. Hirakida, H. Kouno, J. Takahashi, and M. Yahiro, Phys. Rev. D 94, 014011 (2016).

[47] P. de Forcrand, Proc. Sci. LAT2009 (2009) 010 [arXiv: 1005.0539].

[48] M. Matsumoto and T. Nishimura, ACM Trans. Model. Comput. Simul. 8, 3 (1998).

[49] J. Engels, S. Mashkevich, T. Scheideler, and G. Zinovev, Phys. Lett. B 365, 219 (1996).

[50] Z. Fodor and S. Katz, Phys. Lett. B 534, 87 (2002).

[51] Z. Fodor, S. Katz, and K. Szabo, Phys. Lett. B 568, 73 (2003).

[52] Z. Fodor and S. Katz, J. High Energy Phys. 03 (2002) 014.
[53] Z. Fodor and S. Katz, J. High Energy Phys. 04 (2004) 050.

[54] P. de Forcrand and T. Rindlisbacher, EPJ Web Conf. 175, 07026 (2018).

[55] A. Roberge and N. Weiss, Nucl. Phys. B275, 734 (1986).

[56] M. D'Elia and M.-P. Lombardo, Phys. Rev. D 67, 014505 (2003).

[57] P. de Forcrand and O. Philipsen, Nucl. Phys. B642, 290 (2002).

[58] P. de Forcrand and O. Philipsen, Nucl. Phys. B673, 170 (2003).

[59] M. D'Elia and M.-P. Lombardo, Phys. Rev. D 70, 074509 (2004).

[60] L. D. McLerran and B. Svetitsky, Phys. Rev. D 24, 450 (1981). 